\title{
Comments on Mielnik's Generalized (Non Linear) Quantum Mechanics *
}

\author{
Rudolf Haag and Ulrich Bannier \\ II. Institut für Theoretische Physik der Universität Hamburg, D-2000 Hamburg 50, \\ Federal Republic of Germany
}

\begin{abstract}
We discuss a model of non linear quantum mechanics in which the wave equation satisfies the homogeneity condition (2.1). It is argued that in this model the set of (mixed) states is a simplex.
\end{abstract}

\section{The Setting}

The term "state" of a system is customarily used in quantum physics for a statistical ensemble of (equally prepared) samples of the system. Since such ensembles may always be mixed the set $\mathscr{S}$ of states is a convex set, i.e. with $\omega_{1}, \omega_{2} \in \mathscr{S}$ and $0<\lambda<1$

$$
\omega=\lambda \omega_{1}+(1-\lambda) \omega_{2}
$$

is again a state, namely the mixture of $\omega_{1}$ and $\omega_{2}$ with weights $\lambda$ and $(1-\lambda)$. The extremal points of the convex set $\mathscr{S}$ (i.e. those states $\omega$ which cannot be represented as a mixture of others) are the pure states. We denote their set by $\mathscr{E}$. Since we shall be concerned here with the simplest possible generalization of ordinary quantum mechanics it suffices to consider the case where $\mathscr{S}$ is "atomic" i.e. where every state $\omega$ is a countable convex combination of pure states ${ }^{1}$

$$
\omega=\sum \lambda_{i} \Phi_{i}, \quad \Phi_{i} \in \mathscr{E}, \quad \lambda_{i}>0, \quad \sum \lambda_{i}=1 .
$$

Still $\mathscr{E}$ will not determine $\mathscr{S}$ because in general different mixtures of pure states may result in ensembles which are indistinguishable by any measurement. The limitations in observability introduce an equivalence relation (denoted by $\sim$ ) in

Dedicated to Professor Günther Ludwig on the occasion of his sixtieth birthday

More generally one might take $\mathscr{E}$ to be a measure space and replace (1.2) by

$$
\omega=\int_{\mathscr{E}} \Phi d \mu(\Phi)
$$

with $\mu$ a (positive, normalized) measure on $\mathscr{E}$ 
the collection $\mathscr{C}$ of convex combinations of pure states. A state is then an equivalence class in $\mathscr{C}$; symbolically

$$
\mathscr{S}=\mathscr{C} / \sim \text {. }
$$

If there is no (nontrivial) equivalence relation i.e. if the classes consist of but one element then $\mathscr{S}$ is a simplex. This is the situation in classical physics ${ }^{2}$. In general the structure of $\mathscr{S}$ as a convex set (the "statistical figure" in the terminology of [1]) determines the nature of the observational conclusions which can be made about the system, in particular the "quantum logic". To fix this we have to describe $\mathscr{E}$ and the equivalence relation $\sim$.

Let us now consider the case where the system is a single spinless particle and where as in Schrödinger's wave mechanics a pure state $\Phi$ is described by a complex valued wave function $\varphi(x)$. Again we do not assume that the correspondence between a pure state $\Phi$ and wave function $\varphi$ is one to one but rather that $\Phi$ corresponds to an equivalence class of such functions. In order to determine the equivalence relation (in the space of wave functions as well as in $\mathscr{C}$ ) we have to specify what observations are possible. Following [1] we assume that the basic observation is a measurement of the position of the particle. All other possible observations are obtained by letting the particle move for a certain time interval under the influence of some external fields (which can be chosen and varied by the experimenter) and making a position measurement at the end of this time interval. If we denote the external fields symbolically by $A$ then the wave function is transformed in the time $t$ (following the preparation of the ensemble) to a wave function (Schrödinger picture)

$$
\varphi_{A}=T_{A}(t) \varphi,
$$

where $T_{a}(t)$ is some operator acting in the space of wave functions.

To complete the description of the general scheme we have to specify

i) the expression for the probability $p(\Delta, \varphi)$ of finding a particle of the pure ensemble represented by the wave function $\varphi$ in the space-region $\Delta$,

ii) the possible external agents $A$ which determine the set of obtainable operators $T_{A}(t)$ (generating the "motion group" in the terminology of [1]).

For ii) Mielnik suggests that the Schrödinger equation might be rep̀laced by a nonlinear wave equation and proposes some examples which as in the case of ordinary quantum mechanics lead to a continuity equation for a suitably defined probability density $\varrho(x)$. Then i) may be answered by taking

$$
p(\Delta, \varphi)=\int_{\Delta} \varrho_{\varphi}(x) d^{3} x .
$$

We mention here two of the proposals of [1].

$$
\text { a) } i \hbar \frac{\partial \varphi}{\partial t}=-\frac{\hbar^{2}}{2 m} \Delta\left(|\varphi|^{2} \varphi\right)+V|\varphi|^{2} \varphi \text {. }
$$

The external agent which can be chosen by the experimenter is a scalar potential $V(\boldsymbol{x}, t)$. Here one has a continuity equation for $\varrho=|\varphi|^{4}$. One would then put

$$
p(\Delta, \varphi)=\frac{1}{N} \int_{\Delta}|\varphi|^{4} d^{3} x
$$

2 Of course the restriction to countable convex combinations of pure states would not be reasonable there any more 
where $N$ is a normalization factor (see below),

$$
\text { b) } i \hbar \frac{\partial \varphi}{\partial t}=-\frac{\hbar^{2}}{2 m} \Delta \varphi+V \varphi+U|\varphi|^{2} \varphi \text {. }
$$

Here the $L^{2}$-norm is conserved and one puts

$$
p(\Delta, \varphi)=\frac{1}{N} \int_{\Delta}|\varphi|^{2} d^{3} x .
$$

The external agents available are the two scalar potentials $V(\boldsymbol{x}, t), U(\boldsymbol{x}, t)$. If one does not make use of $U$ one has ordinary wave mechanics so that this scheme is an extension of Schrödinger theory.

\section{Homogeneity}

The wave Equations (1.6) and (1.8) do not satisfy the homogeneity condition ${ }^{3}$

$$
\frac{\partial}{\partial t}(c \varphi)=c \frac{\partial \varphi}{\partial t}
$$

for $c \neq 1$. This affects the question of normalization.

Let us consider the case b) which is simpler to discuss and also more realistic. The total probability of finding the particle at a given time somewhere in space should be equal to 1 :

$$
p\left(\mathbb{R}^{3}, \varphi\right)=\frac{1}{N}\|\varphi\|^{2}=1 ; \quad \text { so } \quad N=\|\varphi\|^{2} .
$$

Unlike ordinary quantum mechanics and due to the fact that (2.1) is not satisfied we can determine $\left\|_{\varphi}\right\|$ already from the ratios of the counting rates for different experiments, disregarding (2.2). To find $\varphi$ at $t=0$ we can, using first only ordinary quantum mechanical potentials $V$ (taking $U=0$ ) determine the shape of the wave function from the ratios of counting rates i.e. determine $\varphi$ up to an arbitrary complex factor $c$. If, on the other hand, we use a "strange" potential $U$ for some time which changes $\varphi$ by a nonlinear operator $T$ to $T \varphi$ then if the homogeneity condition $T(c \varphi)=c T \varphi$ is not satisfied the shape of the wave function $T \varphi$ will depend on the absolute value of $c$ so that also $|c|$ and therefore $\|\varphi\|$ can be determined from ratios of counting rates. Therefore $\varphi$ and $\lambda \varphi$ are different pure states. One might think of imposing the additional condition

$$
\|\varphi\|=1
$$

in order to bring the pure states again into one to one correspondence with unit rays in Hilbert space. But this is not admissable due to macroscopic locality. Consider a sequence of wave functions

$$
\varphi_{n}=\psi+\psi_{n}^{\prime}
$$

where the support of $\psi_{n}^{\prime}$ is separated from that of $\psi$ by a distance $r_{n}$ and $\lim _{n \rightarrow \infty} r_{n} \rightarrow \infty$. Let $T$ be any operator in the motion group resulting from the action

3 More precisely: if $\varphi(x, t)$ is a solution and $c$ a constant the $c \varphi$ is not a solution 
of potentials $U, V$ during a finite time interval $t$ (the choice of $U, V, t$ being arbitrary but fixed i.e. independent of $n$ ). The fact that the evolution law is a differential equation implies presumably

$$
\lim _{n \rightarrow \infty}\left\|T \varphi_{n}-T \psi-T \psi_{n}^{\prime}\right\|=0
$$

and, for any fixed, bounded $\Delta$

$$
\lim _{n \rightarrow \infty} \int_{\Delta}\left|T \psi_{n}^{\prime}\right|^{2} d^{3} x=0 .
$$

Thus for $n \rightarrow \infty$ the ensemble $\varphi_{n}$ will give the same ratios of counting rates as the ensemble described by $\psi$ for all experiments performed in finite space time regions. Choosing $\left\|_{\varphi_{n}}\right\|^{2}=1$ the probabilities are scaled

$$
p\left(\Delta, T \varphi_{n}\right) \rightarrow\|\psi\|^{2} p(\Delta, T \psi)
$$

which means that in the ensemble $\varphi_{n}$ the fraction $\left(1-\left\|\psi^{2}\right\|^{2}\right)$ of systems escapes the detection and the remainder behaves like an ensemble with wave function $\psi$. This shows that a state corresponding to a wave function $\psi$ with $\|\psi\|<1$ can indeed be prepared [essentially by preparing a state $\varphi_{n}$ of the form (2.4) with $\left\|\varphi_{n}\right\|=1$ and absorbing the part which is far away].

To sum up: The violation of the homogeneity condition (2.1) has the consequence that wave functions with the same shape but differing in norm represent different pure states. The number $\|\varphi\|$ is a constant of motion which although related to the conservation of the number of systems in the ensemble is not a normalization factor but influences the ratios of counting rates. This is not a decisive objection against such models but at this stage it appears perhaps more natural to consider wave equations which - though non linear-satisfy the homogeneity condition (2.1). An example which we will discuss somewhat in the next section is

$$
i \frac{\partial \varphi}{\partial t}=-\Delta \varphi+V \varphi+\boldsymbol{A} \cdot \nabla S_{\varphi} \varphi \quad\left(\operatorname{set} \operatorname{ting} \hbar=1, m=\frac{1}{2}\right)
$$

where $\nabla S_{\varphi}$ is the gradient of the phase of $\varphi$ i.e.

$$
\nabla S_{\varphi}=\frac{\varphi^{*} \nabla \varphi-\varphi \nabla \varphi^{*}}{2 i|\varphi|^{2}} .
$$

The external agents are a scalar potential $V(\boldsymbol{x}, t)$ and vector potential $\boldsymbol{A}(\boldsymbol{x}, t)$.

\section{The Statistical Figure in the Model (2.7)}

The evolution equation proposed in (2.7) is non linear but homogeneous. Therefore as in Schrödinger theory the pure states are in one to one correspondence with rays in $L^{2}$. The availability of the "vector potential" $\boldsymbol{A}$ as a possible external agent makes a finer distinction of mixed ensembles possible. In fact we want to show

Proposition 1. If $\Phi_{i}(i=1,2, \ldots, n)$ and $\Phi_{j}^{\prime}\left(j=1,2, \ldots, n^{\prime}\right)$ are two different sets of pure states then two ensembles $\omega=\sum \lambda_{i} \Phi_{i}$ and $\omega^{\prime}=\sum \lambda_{j}^{\prime} \Phi_{j}^{\prime}$ are always distinguishable (inequivalent). 
This means that (up to topological questions which we do not study here) the statistical figure is a simplex.

Proof. If $\omega \sim \omega^{\prime}$ then at least the corresponding density matrices must be identical; otherwise we could already distinguish $\omega$ and $\omega^{\prime}$ by measurements using only the ordinary quantum mechanical potentials $V$.

Let $\varphi_{k}\left(k=1,2, \ldots, n+n^{\prime}\right)$ be wave functions representing respectively. $\Phi_{i}$ for $(k=i=1, \ldots, n)$ and $\Phi_{j}^{\prime}$ for $\left(k=n+j, j=1, \ldots, n^{\prime}\right)$, and $\mu_{k}$ real numbers $\mu_{k}=\lambda_{k}$ (for $k=i$ ), $\mu_{k}=-\lambda_{j}$ (for $k=n+j$ ). Then, if $\omega \sim \omega^{\prime}$ we must have

$$
\sum_{k} \mu_{k} \overline{\left(T \varphi_{k}\right)\left(\boldsymbol{x}^{\prime}\right)}\left(T \varphi_{k}\right)(\boldsymbol{x})=0
$$

for any operator $T$ of the motion group ${ }^{4}$. Using (3.1) for $T=1$ and for $T$ corresponding to a motion (2.7) for an infinitesimal time interval we get

$$
\begin{aligned}
& \sum_{k} \mu_{k} \overline{\varphi_{k}\left(\boldsymbol{x}^{\prime}\right)} \varphi_{k}(\boldsymbol{x})=0, \\
& \sum_{k} \mu_{k} \overline{\varphi_{k}\left(\boldsymbol{x}^{\prime}\right)} \varphi_{k}(\boldsymbol{x})\left(\left(\nabla S_{k}\left(\boldsymbol{x}^{\prime}\right) \boldsymbol{A}\left(\boldsymbol{x}^{\prime}\right)-\nabla S_{k}(\boldsymbol{x}) \boldsymbol{A}(\boldsymbol{x})\right)=0 .\right.
\end{aligned}
$$

Since the function $\boldsymbol{A}(\boldsymbol{x})$ is free to choose we must have

$$
\sum_{k} \mu_{k} \overline{\varphi_{k}\left(\boldsymbol{x}^{\prime}\right)} \varphi_{k}(\boldsymbol{x}) \nabla S_{k}(\boldsymbol{x})=0 \text {. }
$$

Suppose now that the $\varphi_{k}$ span an $m$-dimensional subspace (certainly $m<n+n^{\prime}$ since the wave functions cannot be linearly independent if $\left.\omega \sim \omega^{\prime}\right)$ and choose an orthonormal basis $\chi_{r}(r=1,2, \ldots, m)$ in this space. Then

$$
\varphi_{k}=\sum_{r} c_{k r} \chi_{r}
$$

and (3.2) and (3.4) become

$$
\begin{aligned}
& \sum_{k=1}^{n+n^{\prime}} \mu_{k} \bar{c}_{k r} c_{k r^{\prime}}=0 \quad \text { for any } r, r^{\prime}, \\
& \sum_{k=1}^{n+n^{\prime}} \mu_{k} \bar{c}_{k r} \varphi_{k}(\boldsymbol{x}) \nabla S_{k}(\boldsymbol{x})=0 \text { for any } r, \boldsymbol{x} .
\end{aligned}
$$

We may assume that the $\varphi_{i}, \varphi_{j}^{\prime}$ are a minimal set of wave functions for which the equivalence $\omega \sim \omega^{\prime}$ holds i.e. that on the left hand side of (3.6) no partial sum over a proper subset $I$ of indices $k$ vanishes. In particular we may choose the basis so that

$$
\sum_{k \in I} \mu_{k} \bar{c}_{k 1} c_{k 1}=0 \quad \text { implies } \quad I=\left\{1,2, \ldots, n+n^{\prime}\right\}
$$

and $\operatorname{Im} \bar{c}_{k 1} c_{k 2} \neq 0$ for all $k=1,2, \ldots, n+n^{\prime}$.

The essential point is now that for given expansion coefficients $c_{k r}$ the phases $S_{k}(x)$ depend on the basis functions $\chi_{r}$. Since already the ordinary quantum mechanical motions $(\boldsymbol{A}=0)$ generate the unitary group (compare [1]) we may choose for $T$ in (3.1) in particular an arbitrary unitary operator. This means in turn that (3.7) should hold for arbitrary choice of the orthonormal basis functions $\chi_{r}(x)$.

Choose now $\chi_{1}$ and $\chi_{2}$ to have the same support, e.g. the cube $K$ with the origin as center and edge length 1 and choose the support of $\chi_{r}$ with $r>2$ disjoint from $K$.

4 The left hand side is the difference between the statistical matrices (in the $\boldsymbol{x}$-representation) which correspond to $\omega$ and $\omega^{\prime}$ after a motion $T$ 
Specifically let

$$
\begin{aligned}
& \chi_{1}(\boldsymbol{x})= \begin{cases}1 & \text { for } \quad x \in K \\
0 & \text { otherwise },\end{cases} \\
& \chi_{2}(\boldsymbol{x})=\operatorname{ax} \chi_{1}(\boldsymbol{x}), \quad a=2 \sqrt{3} .
\end{aligned}
$$

Then for $\boldsymbol{x} \in K$

$$
\begin{aligned}
\varphi_{k}(\boldsymbol{x}) & =c_{k 1}+a c_{k 2} x \\
\nabla S_{k}(\boldsymbol{x}) & =\frac{\operatorname{Im} \overline{\varphi_{k}(\boldsymbol{x})}\left(\nabla \varphi_{k}(\boldsymbol{x})\right)}{\left|\varphi_{k}(\boldsymbol{x})\right|^{2}} \\
& =\frac{a \operatorname{Im} \bar{c}_{k 1} c_{k 2}}{\left|c_{k 1}\right|^{2}+2 a x \operatorname{Re} \bar{c}_{k 1} c_{k 2}+a^{2} x^{2}\left|c_{k 2}\right|^{2}} .
\end{aligned}
$$

Condition (3.7) demands now that the functions $\varphi_{k} \nabla S_{k}$ should be linearly dependent. According to (3.11) and (3.12) these are broken rational functions and only those of them can be linearly dependent, for which the positions of the poles coincide. Now the poles of $\nabla S_{k}$ lie at $-\frac{\bar{c}_{k 1}}{a \bar{c}_{k 2}}$. Therefore we can have linear dependence only for such subsets of $\varphi_{k} \nabla S_{k}$ for which the $\varphi_{k}$ within $K$ are proportional and hence the $\nabla S_{k}$ identical. In that case we get from (3.7) for such a subset $I$ of indices $k$

$$
\sum_{k \in I} \mu_{k} \bar{c}_{k r} \varphi_{k}(x)=0, \quad x \in K
$$

or

$$
\sum_{k \in I} \mu_{k} \bar{c}_{k r} c_{k r^{\prime}}=0, \quad r, r^{\prime}=1,2 .
$$

Due to the minimality assumption (3.8) this means that $I=\left\{1,2, \ldots, n+n^{\prime}\right\}$ i.e. that all functions $\varphi_{k}$ must be proportional within $K$ which in turn implies that the subspace spanned by them is at most $(m-1)$-dimensional which is a contradiction.

Thus the fact that we can influence the motion by the non linear $\boldsymbol{A}$-term in this model makes mixtures of different sets of pure states distinguishable. As far as only single particle systems are considered one might claim that $|\varphi(\boldsymbol{x})|$ and $\nabla S(\boldsymbol{x})$ describe the "objective state" of an individual particle. However it is not our intention to maintain that thereby one achieves a classical theory (where $|\varphi|$ and $\nabla S$ are hidden variables of the particle) because problems with such an interpretation would immediately arise as soon as one considers 2-particle systems.

\section{Reference}

1. Mielnik, B.: Commun. math. Phys. 37, 221-256 (1974)

Communicated by R. Haag

Received January 8, 1978 